\title{
Trochlear Nerve Palsy Associated with Claude Bernard-Horner Syndrome after Brainstem Stroke
}

\author{
Rodrigo Bazan Gabriel Pereira Braga \\ Daniela Laranja Gomes Seizo Yamashita \\ Luiz Eduardo Betting Luiz Antonio de Lima Resende
}

Departamento de Neurologia, Psiquiatria e Psicologia, Faculdade de Medicina de Botucatu - UNESP - Univ Estadual Paulista, Botucatu, Brazil

\section{Key Words}

Trochlear nerve $\cdot$ Claude Bernard-Horner syndrome $\cdot$ Ocular palsy $\cdot$ Stroke

\begin{abstract}
The association of unilateral trochlear nerve palsy with Claude Bernard-Horner syndrome represents a rare clinical condition. We present the case of a patient with this unusual presentation. The investigation performed implicated cerebrovascular disease as the underlying cause of the condition in this patient.
\end{abstract}

\section{Introduction}

Trochlear nerve palsy may be clinically characterized by vertical diplopia, incomitant hypertropia that increases upon head tilt toward the paralyzed site (positive Bielschowsky's test), excyclotropia, and head tilt [1]. The differentiation between acutely acquired palsy vs. the decompensation of a congenital palsy is important for an accurate diagnosis [1]. The presence of acute unilateral trochlear nerve palsy with Claude BernardHorner syndrome represents a rare clinical condition [2]. In a review of 215 cases of trochlear nerve palsy examined over a period of 23 years, only 5 patients presented with this condition [2]. The current report describes a patient with trochlear nerve palsy associated with Claude Bernard-Horner syndrome and contralateral hemiparesis, with onset following a hypertensive crisis. 


\section{Case Report}

A 35-year-old Caucasian woman with a history of chronic headache and systemic arterial hypertension was admitted with sudden acute intense bitemporal headache, diplopia, dysarthria, and left hemiparesis. The patient evolved to torpor within a few hours. The clinical examination showed a blood pressure of 170/110 mm Hg. The patient's blood pressure was lowered with antihypertensive drugs, including sodium nitroprusside, and she regained consciousness the next day. A neurological examination showed right trochlear nerve palsy, slight ipsilateral Claude Bernard-Horner syndrome (fig. 1), and left hemiparesis. At rest, the patient presented cyclovertical diplopia when her head was tilted down and to the right. Magnetic resonance imaging (MRI) of the brainstem and cerebellum, acquired on the sixth day, showed multiple small areas localized mainly to the pons and mesencephalon that were distinguished by T1 and T2 hyperintense signals and no contrast enhancement (fig. 2). The cerebrospinal fluid and investigation for secondary hypertension, infectious and inflammatory diseases were normal. The patient's evolution was satisfactory, with a slow and progressive recovery. An MRI in the fourth week showed remission of the previous lesions; the patient was asymptomatic at that time and returned to her daily activities.

\section{Discussion}

The most frequent causes of trochlear nerve palsy are traumas, surgical injuries, tumors, demyelinating and inflammatory diseases such as meningitis and cysticercosis [2]. Brainstem stroke represents a rare cause of trochlear nerve palsy [3], in which infarcts may be extensive or restricted to the microvascular territory $[4,5]$.

The patient presented in the current report developed sudden-onset clinical manifestations associated with arterial hypertension. These clinical features were suggestive of a vascular etiology, which was supported by the neuroimaging findings (fig. 2). Most likely, small bleedings at the brainstem and cerebellum occurred as consequence of increased blood pressure. This clinical picture has previously been described in patients with trochlear nerve palsy [5-7].

The right-side Claude Bernard-Horner syndrome and left-side hemiparesis observed in this patient may be related to the right-side pons lesion that was observed (fig. 2a). In addition, the right-side trochlear palsy was possibly a result of the left dorsal mesencephalic lesion (fig. 2b, arrow). After exiting the trochlear nucleus, which is ventrolateral to the cerebral aqueduct, the nerve fascicles course posteroinferiorly around the cerebral aqueduct and decussate in the dorsal midbrain $[1,5]$. Therefore, in the current patient, the involvement of the trochlear nerve must have occurred before the decussation, resulting in contralateral palsy. The literature also contains descriptions of contralateral trochlear nerve palsy with ipsilateral Horner's syndrome [8]. Guy et al. [8] describe nuclear or fascicular involvement of the trochlear nerve prior to decussation in the superior medullary velum and impairment of the adjacent sympathetic fibers. In the current report, the multiplicity of lesions observed in the patient likely was responsible for the unusual clinical presentation.

In the case presented here, progressive improvement was observed, and the patient was asymptomatic at the end of the fourth week. Similar outcomes have been described in patients with trochlear nerve palsy related to minor vascular lesions. However, there is not sufficient evidence to draw definitive prognostic conclusions in this rare situation $[3$, $4,6]$. 

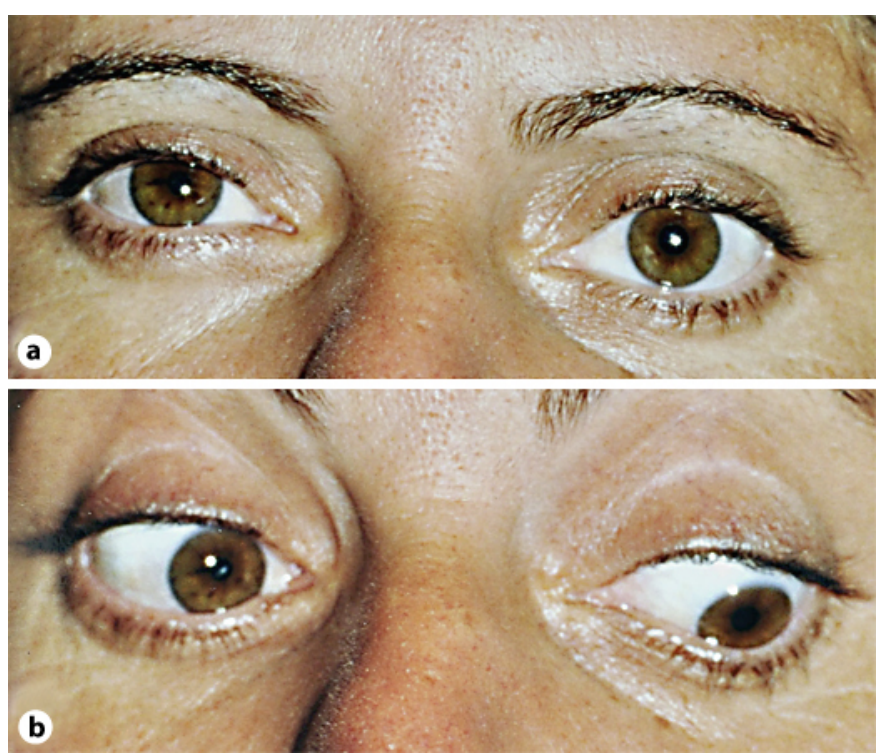

Fig. 1. Trochlear nerve palsy and Claude Bernard-Horner syndrome. a Slight ptosis on the right side compatible with Claude Bernard-Horner syndrome. $\mathbf{b}$ Hypertropia of the right eye when performing left gaze. The hypertropia was increased on head tilt to the right (positive Bielschowsky's test).
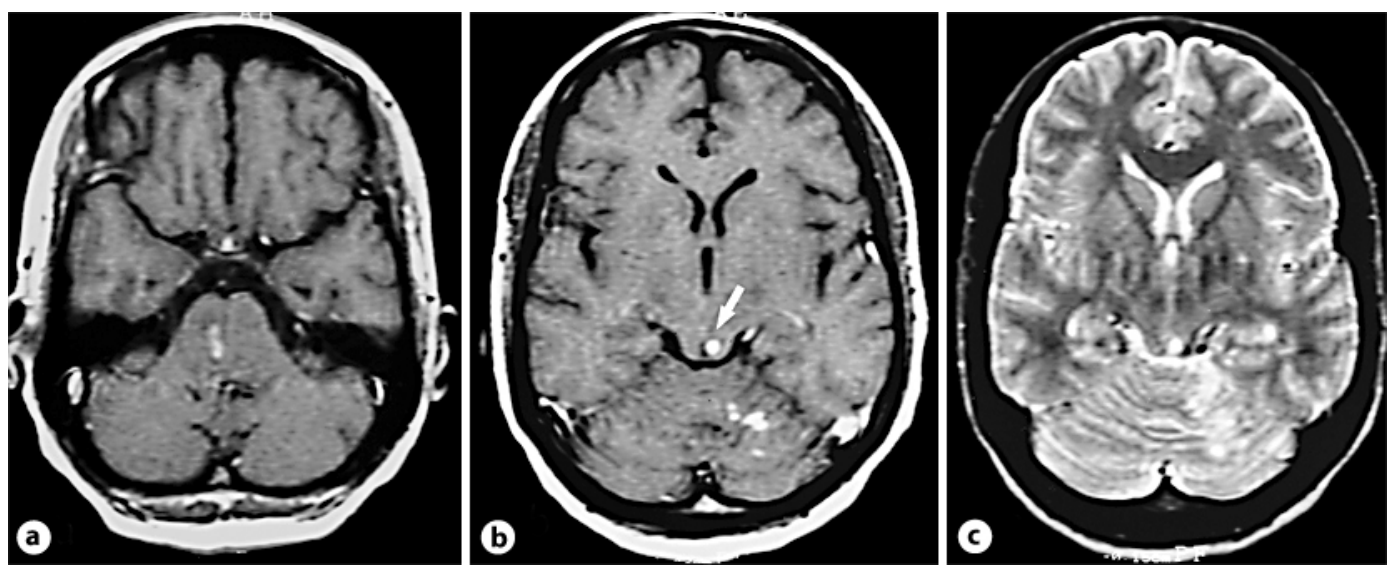

Fig. 2. Magnetic resonance imaging showing multiple hyperintense small areas localized at the brainstem and cerebellum. a Axial T1 post contrast, showing a hyperintense area localized at the pons. b Axial T1 post contrast at a level superior to that shown in a, demonstrating multiple lesions localized at the left cerebellar hemisphere and at the left dorsal mesencephalon. This lesion (arrow) was likely responsible for the clinical manifestations described. c Axial T2 showing the same lesions depicted in $\mathbf{b}$. 


\section{References}

1 Brazis PW, Masdeu JC, Biller J: The localization of lesions affecting the ocular motor system; in Brazis PW, Masdeu JC, Biller J (eds): Localization in Clinical Neurology. Philadelphia, Lippincott Williams and Wilkins, 2007, pp 169-270.

2 Keane JR: Fourth nerve palsy: historical review and study of 215 inpatients. Neurology 1993;43:2439-2443.

-3 Lee SH, Park SW, Kim BC, Kim MK, Cho KH, Kim JS: Isolated trochlear palsy due to midbrain stroke. Clin Neurol Neurosurg 2010;112:68-71.

4 Keane JR: Tectal fourth nerve palsy due to infarction. Arch Neurol 2004;61:280.

5 Makki AA, Newman NJ: A trochlear stroke. Neurology 2005;65:1989.

-6 Papanas N, Heliopoulos I, Piperidou H, Matezos E: Simultaneous, painless, homolateral oculomotor and trochlear nerve palsies in a patient with type 2 diabetes mellitus. Neuropathy or brainstem infarction? Acta Diabetol 2006;43:19-21.

7 Walsh RA, Murphy RP, Moore DP, McCabe DJH: Isolated trochlear infarction. Arch Neurol 2010;67:892-893.

$>8$ Guy J, Day AL, Mickle JP, Schatz NJ: Contralateral trochlear nerve paresis and ipsilateral Horner's syndrome. Am J Ophthalmol 1989;107:73-76. 